\title{
DOENÇAS NEGLIGENCIADAS E O ENSINO DE CIÊNCIAS: REFLEXÕES ELABORADAS A PARTIR DAS PROPOSTAS CURRICULARES
}

\section{NEGLECTED DISEASES AND EDUCATION SCIENCE: REFLECTIONS PRODUCED FROM OF PROPOSALS CURRICULUM}

\author{
Sheila Soares de Assis ${ }^{1}$, Tania Araújo-Jorge² \\ ${ }^{1}$ Fundação Oswaldo Cruz/Instituto Oswaldo Cruz/Laboratório de Inovações, Terapias, Ensino e \\ Bioprodutos (LITEB) - Doutoranda do Programa de Pós Graduação em Ensino em Biociências e Saúde, \\ sheila.assisbiouff@gmail.com \\ ${ }^{2}$ Fundação Oswaldo Cruz/Instituto Oswaldo Cruz/Laboratório de Inovações, Terapias, Ensino e \\ Bioprodutos (LITEB), taniaaj@ioc.fiocruz.br
}

\begin{abstract}
RESUMO
A Organização Mundial da Saúde (OMS) estima que mais de 1 bilhão de pessoas em todo o mundo são acometidas pelas doenças negligenciadas (DN). Estão inclusos nesse grupo os agravos relacionados à pobreza como dengue, leishmanioses, malária, geohelmintoses, entre outros. Como uma das medidas a serem empregadas para o enfrentamento da pobreza no país está a inclusão deste tema nos conteúdos curriculares da educação básica e técnica. O objetivo do estudo foi refletir sobre o tema das doenças negligenciadas nas propostas curriculares de cinco estados brasileiros, sendo cada um pertencente a uma região geográfica do país. A pesquisa tem cunho qualitativo e utilizou-se a análise interpretativa. Verificamos que somente dois dos currículos analisados mencionam as doenças negligenciadas, prevalece ainda o indicativo de normas prescritivas e pouco encorajamento para uma participação autônoma da sociedade nas ações de saúde. A participação de docentes na elaboração dos documentos é percebida como avanço no processo de construção curricular.
\end{abstract}

Palavras-chave: Doenças negligenciadas; ensino de Ciências; currículo; saúde.

\begin{abstract}
The World Health Organization (WHO) estimates that more than 1 billion people worldwide are affected by neglected diseases (ND). This group includes health problems related to poverty such as dengue, leishmaniasis, malaria, helminths, leprosy, among others. Inclusion of this themes in the curricula of basic and technical education helps to face this problem in developing countries. The aim of this study was to identify the presence of and to reflect on the subject of neglected diseases in the curricular proposals in five Brazilian states corresponding to each region and to reflect on possibilities to integrate them at schools. The research has a qualitative approach and an interpretive analysis. We found only two curricula mentioning neglected diseases, prescriptive norms predominates and we saw little encouragement for an autonomous participation of the society in health activities. Participation of teachers in documents conception was perceived as a breakthrough in the curriculum development process.
\end{abstract}

Key words: Neglected diseases; science education, curriculum, health.

\section{INTRODUÇÃO}




\section{Ensino, Saúde e Ambiente - V 7 (1), Edição Especial, maio de 2014}

Dentre os desafios do milénio estabelecidos pela ONU (Organização das Nações Unidas) está incluso o esforço para a redução da pobreza a nível global e consequentemente das doenças negligenciadas (DN) (ONU, 2000). Segundo a Organização Mundial de Saúde (OMS), as DN acometem mais de um bilhão de pessoas em todo o mundo anualmente (OMS, 2010). Compõem o grupo destes agravos as enfermidades infecciosas que apresentam maior prevalência em países em desenvolvimento e outras 17 doenças associadas à pobreza que contribuem para a manutenção do cenário de desigualdade (BRASIL, 2010; MSF, 2001; OMS, 2001; OMS, 2010).

No Brasil, em 2011, com a elaboração do Plano para Erradicação da Pobreza extrema, pelo Ministério do Desenvolvimento Social (MDS) ${ }^{1}$, o Instituto Oswaldo Cruz (IOC/Fiocruz-RJ) emitiu a nota técnica institucional N. ${ }^{\circ}$ 1/2011/IOCFIOCRUZ/DIRETORIA reportando a necessidade de inclusão do tema das DN no na ação desencadeada pelo MDS. A nota técnica sinaliza que a tuberculose, dengue, esquistossomose, hanseníase, malária, geohelmintoses, parasitoses intestinais e anemias carenciais são as principais doenças associadas à pobreza no contexto nacional (IOC, 2011). Segundo este documento, os conteúdos curriculares da educação básica e da educação técnica deveriam contemplar questões e temas relacionados às doenças da pobreza como forma de enfrentamento destes agravos e ampliação da equidade social (IOC, 2011).

\section{ENSINO DE CIÊNCIAS, SAÚDE E CURRÍCULO}

O espaço escolar manifesta-se como instituição de reprodução, proposital ou não, das aspirações da ordem vigente na sociedade no processo de construção de conhecimento. Neste contexto, Krasilchik (2000) aponta que o ensino de Ciências é modificado em função do âmbito político-econômico, tanto nacional como internacional. Até que adquirisse a atual configuração, o ensino de Ciências atravessou diversas transformações e a educação em saúde estruturada no espaço escolar acompanhou estas modificações. Na atual Lei de Diretrizes e Bases da Educação 9.394/96, a educação em saúde não é citada. De tal modo, somente a partir de 1997 e 1998 ela é lembrada. Neste período são estabelecidos os Parâmetros Curriculares Nacionais voltados para o $1^{\circ}-2^{\circ}$ segmentos e $3^{\circ}$ e $4^{\circ}$ segmentos do ensino fundamental,

\footnotetext{
${ }^{1}$ Plano Brasil sem Miséria (http://www.brasilsemmiseria.gov.br/).

Edição Especial com os melhores trabalhos apresentados no IV ENECiências: UFF - 13 a 16 d2 maio de 2014. 


\section{Ensino, Saúde e Ambiente - V 7 (1), Edição Especial, maio de 2014}

respectivamente. A saúde é alocada então como um componente transversal (MOHR, 2009).

No âmbito do ensino, ao longo do tempo, os temas que se referem à saúde, especialmente sobre as doenças negligenciadas, têm sido abordados majoritariamente nas aulas de Ciências e de Biologia (SCHALL, 2010; ASSIS, PIMENTA e SCHALL, 2013a, ASSIS, PIMENTA e SCHALL, 2013b; FRANÇA, 2012; VENTURINE e MOHR, 2013). No contexto escolar, as propostas curriculares são um elemento essencial. Goodson (1995, p. 18) destaca que os currículos são "uma estrutura de conhecimento socialmente apresentado, externo ao conhecedor, a ser por ele dominado". Ele pode ser concebido ainda como um artefato social e cultural, produto de demandas sociais, histórica e contextual. Implica relações de poder, transmite visões de mundo, produz identidades individuais e sociais (BRITO e FREITAS, 2009).

Os currículos não expressam um pensamento neutro e puramente técnico. Pelo contrário, eles expõem o saber que é valorizado e considerado essencial por um grupo hegemônico da sociedade. Assim, alguns temas são privilegiados em detrimento de outros visando à manutenção ou alteração de um contexto social em um dado recorte temporal (SILVA, 1999). Os documentos curriculares trazem elementos próprios do cotidiano escolar e ainda aqueles oriundos de outras instâncias como, por exemplo, a sociedade, o Estado e as políticas que este assume como interessantes por diversos aspectos. Desse modo nem sempre esses documentos são pensados para a construção do bem estar social, mas voltados ao mercado e como resposta ao modelo econômico ou a outros interesses (APPLE, 2002a).

Assim, em um momento histórico em que se busca a superação da pobreza extrema no nosso país, e consequentemente o sobrepujamento das doenças negligenciadas que afetam a população por décadas, analisar os currículos de Ciências é crucial na medida em que nos permite apreciar os indícios sobre as contribuições dessa disciplina para o alcance deste fim e ainda os subsídios para a construção de um cenário de justiça social. Nesse sentido, o objetivo deste trabalho é refletir sobre o tema das doenças negligenciadas nas propostas curriculares de cinco estados brasileiros.

\section{METODOLOGIA}

A pesquisa apresenta caráter qualitativo e utilizou-se da técnica de análise documental (ALVES-MAZZOTTI e GEWANDSZNAJDER, 1998). A técnica pode evidenciar fatos novos ou completar informações já levantadas pelo pesquisador Edição Especial com os melhores trabalhos apresentados no IV ENECiências: UFF - 13 a 16 dß̧ maio de 2014. 


\section{Ensino, Saúde e Ambiente - V 7 (1), Edição Especial, maio de 2014}

(VÍCTORA, KNAUTH e HASSEN, 2000). Seu emprego é útil também com a finalidade exploratória indicando quais aspectos devem ser aprofundados em etapas posteriores da pesquisa (ALVES-MAZZOTTI e GEWANDSZNAJDER, 1998).

Para atender o objetivo proposto neste estudo foram selecionadas cinco propostas curriculares de Ciências destinadas ao segundo segmento do ensino fundamental $\left(6^{\circ}\right.$ ao $\left.9^{\circ}\right)$, sendo que cada documento correspondente a um estado brasileiro pertencente a uma das cinco regiões geográficas do país (Quadro 1). Os arquivos foram recuperados a partir do site das respectivas secretarias estaduais de educação.

As reflexões aqui apresentadas correspondem à parte dos dados de uma tese em desenvolvimento que visa elaborar uma proposta de ensino voltada para a abordagem das doenças negligenciadas em ações intersetoriais e alinhadas com os componentes curriculares de Ciências. Assim, a escolha dos documentos analisados se deu devido à facilidade de acesso das pesquisadoras a estes estados. Garantindo, deste modo, a obtenção de outras informações necessárias em etapas subsequentes da pesquisa.

Após sua coleta, as propostas curriculares foram analisadas observando-se a perspectiva do documento, o indicativo ou não para a abordagem das doenças negligenciadas, as determinações contidas para o tratamento do tema e o conteúdo indicado. Para a apreciação fez-se uso da análise interpretativa. Segundo Severino (1996) este tipo de análise permite tomar uma posição própria a respeito das ideias enunciadas, superar as ideias emitidas e ler nas entrelinhas. Em suma, ao submeter o texto a este tipo de apreciação pode se explorar a fecundidade das ideias expostas e permitir, assim, um diálogo com as ideias do autor e com outras que podem ser convergentes ou conflitantes com as explicitadas no material analisado.

Quadro 1: Propostas curriculares de Ciências analisadas.

\begin{tabular}{|c|c|c|c|c|}
\hline & & & & \\
\hline Região & $\begin{array}{l}\text { Estado } \\
\text { Selecionado }\end{array}$ & $\begin{array}{l}\text { Data de } \\
\text { produção/edição }\end{array}$ & Fonte & $\begin{array}{l}\text { Data de } \\
\text { Acesso }\end{array}$ \\
\hline Norte & Acre & 2010 & $\begin{array}{l}\text { http://www.see.ac.gov. } \\
\underline{\text { br/portal/index.php?op }} \\
\underline{\text { tion=com_content\&vie }} \\
\underline{\text { w=article\&id=532\&Ite }} \\
\underline{\text { mid=275 }}\end{array}$ & $27 / 12 / 2013$ \\
\hline
\end{tabular}

Edição Especial com os melhores trabalhos apresentados no IV ENECiências: UFF - 13 a 16 d4 maio de 2014. 
Ensino, Saúde e Ambiente - V 7 (1), Edição Especial, maio de 2014

\begin{tabular}{|c|c|c|c|c|}
\hline Nordeste & Pernambuco & 2013 & $\begin{array}{l}\text { http://www.educacao.p } \\
\text { e.gov.br/portal/?pag=1 } \\
\underline{\text { \&cat }=36 \& a r t=1047}\end{array}$ & $30 / 11 / 2013$ \\
\hline Sudeste & Rio de Janeiro & 2012 & $\begin{array}{l}\text { http://www.rj.gov.br/w } \\
\text { eb/seeduc/exibeconteu } \\
\underline{\text { do?article-id=759820 }}\end{array}$ & $15 / 12 / 2013$ \\
\hline $\begin{array}{l}\text { Centro- } \\
\text { Oeste }\end{array}$ & $\begin{array}{l}\text { Mato Grosso do } \\
\text { Sul }\end{array}$ & 2012 & $\begin{array}{l}\text { http://sistemas.sed.ms. } \\
\text { gov.br/arquivos/refere } \\
\text { ncial_curricular_ensin } \\
\text { o_fundamental.pdf }\end{array}$ & $01 / 12 / 2013$ \\
\hline Sul & Paraná & 2008 & $\begin{array}{l}\underline{\text { http://www.educadores }} \\
\underline{\text { diaadia.pr.gov.br/mod }} \\
\underline{\text { ules/conteudo/conteud }} \\
\text { o.php?conteudo=1 }\end{array}$ & $01 / 12 / 2013$ \\
\hline
\end{tabular}

\section{RESULTADOS E DISCUSSÃO}

As cinco propostas curriculares foram localizadas facilmente a partir do site das respectivas secretárias estaduais de educação. Todos os textos estão expostos de forma pública e não em sítios restritos. Alocar os documentos dessa forma é importante para garantir que não somente os professores pertencentes à rede, mas também pesquisadores da área e a sociedade em geral possam acessá-los, como fizemos.

Embora os textos visem à orientação e direcionamento dos conteúdos a serem tratados pelo professor não é sempre que este profissional ganha voz em sua produção. A proposta curricular do Rio de Janeiro, por exemplo, foi elaborada por um grupo de pesquisadores de uma Universidade Federal e apesar de descrever que houve reuniões com os profissionais da rede o texto deixa claro que as sugestões destes só foram incorporadas quando possível. A construção curricular de forma democrática e não polarizada é uma necessidade e uma demanda antiga dos profissionais da educação e pode-se perceber como avanço a abertura para a participação da classe nos processos de construção. Ainda que na prática esta ação seja tímida, verificada em apenas duas propostas.

Todos os documentos apresentam uma preocupação em viabilizar uma melhor aprendizagem aos estudantes, porém apenas os currículos do estado do Rio de Janeiro e do Paraná deixam claro, em seu texto introdutório, a finalidade de apropriação do Edição Especial com os melhores trabalhos apresentados no IV ENECiências: UFF - 13 a 16 dई maio de 2014. 


\section{Ensino, Saúde e Ambiente - V 7 (1), Edição Especial, maio de 2014}

conhecimento científico e a inserção destes na prática social dos alunos. Esta perspectiva para a construção curricular é essencial quando tratamos de temas relacionados à saúde, especialmente sobre as doenças negligenciadas, ou temáticas que mobilizam valores de cunho social, econômico, ambiental, entre outros. É recorrente a prática de separação entre o biológico e o social quando se aborda a temática das doenças negligenciadas no ensino de Ciências e a invisibilidade do tema na prática pedagógica tem sido criticado em estudos do campo que advogam a favor de uma percepção ampliada da saúde e do ambiente e que buscam um caráter que se aproxime da promoção da saúde (MOHR, 2002; KRASILCHIK e MARANDINO, 2007; ASSIS, PIMENTA e SCHALL, 2013a; FRANÇA, MARGONARI, SCHALL, 2013).

Observa-se que das cinco propostas três foram elaboradas após a divulgação da nota técnica do Instituto Oswaldo Cruz (IOC/Fiocruz), reportada anteriormente, que indica a incorporação do tema nos currículos do ensino básico e chama a atenção das esferas governamentais sobre a emergência do tema no espaço escolar. Entretanto, as doenças negligenciadas são reportadas apenas nas propostas curriculares do Rio de Janeiro e do Mato Grosso do Sul. O currículo mínimo do estado do Rio de Janeiro indica a abordagem de doenças como a dengue e o cólera no terceiro bimestre do $7^{\circ}$ ano. Embora ambas sejam doenças negligencias deve-se estar atento às doenças de maior emergência no contexto nacional e regional. Historicamente o Rio de Janeiro tem experimentado epidemias sucessivas de dengue e somente em 2013 foram 10.765 casos confirmados da doença no estado (BRASIL, 2014), justificando assim a necessidade de abordagem do tema. Contudo, o cólera que é tratado como um conteúdo prioritário no documento não tem apresentado incidência relevante no país, ao contrário de leishmanioses e outras DN, por exemplo. Segundo o Ministério da Saúde foi registrado um único caso de cólera em São Paulo em 2011 (BRASIL, 2014). A proposta curricular do Mato Grosso do Sul indica como prioritário a abordagem de um conjunto de doenças negligenciadas para os alunos do $7^{\circ}$ ano. Dentre os agravos listados estão AIDS, dengue, doença de Chagas, leishmaniose e leptospirose. Segundo o Ministério da Saúde, somente no Mato Grosso do Sul em 2013 foram confirmados 2. 648.532 casos de AIDS, 8.291 de dengue, 182 casos de leishmanioses, um de leptospirose e não houve notificações de casos novos de doença de Chagas no estado (BRASIL, 2014). Os estados do Acre, Pernambuco e Paraná não comentam e nem recomendam a abordagem de nenhuma doença negligenciada, especificamente, em seus documentos, apesar de 


\section{Ensino, Saúde e Ambiente - V 7 (1), Edição Especial, maio de 2014}

Pernambuco ser o único estado com um Programa específico de controle de sete DN (projeto SANAR, para tracoma, doença de Chagas, hanseníase, filariose, esquistossomose, helmintíase e tuberculose. Deste modo, indica-se um desalinhamento entre políticas públicas de saúde e de educação.

Os dois currículos descritos manifestam a intenção de formação de sujeitos capazes de interferir sobre agravos que atingem as respectivas regiões. Entretanto, devese estar atento a este esforço contínuo no sentido de formar uma massa de sujeitos ativos e engajados socialmente na mitigação de agravos. Nesta perspectiva, podemos refletir sobre o ideal hegemônico ${ }^{2}$ de uma atuação mínima do estado em relação aos aspectos que afetam a saúde e o bem estar da população. Além disso, educadores engajados nas constantes discussões sobre "o que é bom” e "o que deve ser ensinado" poucas vezes refletem profundamente em relação às raízes ideológicas de determinado conteúdo. Assim, mesmo dotados de uma visão crítica, é possível manter uma base dominante (APPLE, 2002b). Sendo assim não basta que o currículo expresse a necessidade de se tratar o tema das doenças negligenciadas, mas deve se atentar as indicações que são relacionadas a elas.

Apesar dos documentos analisados, em seu texto introdutório, reportarem que a saúde deva ser tratada de forma holística e contextualizada, observa-se, ao longo do documento, uma contradição nas propostas que indicaram as doenças negligenciadas e naquelas que se restringiram em apenas indicar a abordagem da saúde. Nos textos oriundos do Rio de Janeiro e do Mato Grosso do Sul os agravos são correlacionados direta e restritamente a aspectos biológicos como corpo humano e microrganismos. Já os outros documentos indicam uma abordagem descontextualizada como, por exemplo, a proposta curricular do Acre que aloca o conteúdo para o $9^{\circ}$ onde são tratados os conteúdos de Química e Física. Segundo Goodson (1995) esse desajuste e descontinuidade dos conteúdos curriculares é um problema recorrente. Além disso, esta situação pode ser agravada, pois é comum haver queixas de professores sobre a dificuldade de implementar o enfoque interdisciplinar e contextualizar o conteúdo. Ou seja, se a própria proposta curricular não estimula tal fim dificilmente este objetivo será alcançado (REZENDE, LOPES e EGG, 2004). Além disso, o forte componente de determinantes sociais da saúde para o entendimento da dinâmica das DN que modela os

\footnotetext{
${ }^{2}$ Segundo Apple (2006) hegemonia é um conjunto organizado de significados e práticas, ao sistema central, eficaz e dominante de significados, valores e ações que são vividos de modo sutil.

Edição Especial com os melhores trabalhos apresentados no IV ENECiências: UFF - 13 a 16 dq maio de 2014. 


\section{Ensino, Saúde e Ambiente - V 7 (1), Edição Especial, maio de 2014}

determinantes biomédicos, também pode permitir sua abordagem em outras disciplinas, como Geografia, História, Português ou Matemática, além de Ciências e Biologia (AAGAARD-HANSEN e CHAIGNAT, 2010).

Há ainda um descompasso entre as formulações teóricas, os problemas de ensino/aprendizagem e questões sociais concretas (GOODSON, 1995). Logo, o que é descrito no currículo podem ser metas inalcançáveis ou que não atendem a real demanda de certo grupo. Dentro das práticas de educação em saúde, estabelecidas no espaço escolar, é comum observar que elas refletem estratégias normativas e prescritivas. Os currículos escolares, por sua vez, acabam sendo um instrumento para este fim quando reproduzem discursos midiáticos ou se abstém de colocar em pauta problemas encontrados nas comunidades a fim de autonomizar os indivíduos (MOHR, 2009).

A escola é um espaço privilegiado de legitimação de alguns saberes em detrimento de outros, sendo que os conhecimentos científicos ali apresentados precisam reter algum diálogo com o cotidiano e os saberes populares, por mais que isso seja complexo (LOPES, 1993). Refletindo sobre como a educação em saúde tem sido expressa no currículo, Mohr (2009) advoga a favor de uma proposta autônoma em que a preocupação central deva ser em capacitar o indivíduo para que ele aja de forma a assegurar a saúde individual ou coletiva, se assim ele desejar. Ou seja, a decisão do agir é do indivíduo e o currículo deve ser um artefato que oriente essa formação e não como mais um instrumento que atua a favor da dominação, propagando ideias impositivas e isentando o Estado de suas responsabilidades.

Apesar de em alguns momentos os textos analisados expressarem que asseguram a autonomia dos professores isso acaba sendo conflitante quando apresentam uma estrutura que indica exatamente "o que" e "quando" o docente deve abordar em suas classes não considerando o dinamismo das salas de aula e as peculiaridades das diferentes comunidades escolares. Essa estrutura rígida acaba por aprisionar professores, alunos e todo o corpo pedagógico como se fosse uma verdadeira grade, a grade curricular (ARROYO, 1988).

\section{CONSIDERAÇÕES FINAIS}

De acordo com a análise do estudo, verificamos que a menção para a abordagem das doenças negligenciadas nas aulas de Ciências ainda é incipiente, pois somente dois dos cinco currículos analisados explicitam que o tema deve ser tratado. Edição Especial com os melhores trabalhos apresentados no IV ENECiências: UFF - 13 a 16 d8 maio de 2014. 
Percebemos uma tentativa de promover o engajamento dos professores na construção dos documentos, ainda que tímida. Contudo, o poder de decisão sobre o conteúdo a ser abordado nas aulas de Ciências parece ainda estar centrado nas mãos de pequenos grupos com responsabilidades executivas. Além disso, o tema também é delegado aos livros didáticos e materiais educativos que podem apresentar uma forma normativa. Os textos ainda apresentam uma maior preocupação em citar doenças ou indicar normas prescritivas relacionadas aos agravos do que os correlacionar com os fatores que os geram e os ciclos que os mantém. Deste modo, parece não se buscar uma formação que vise uma participação ativa e autônoma, ainda que o discurso possa apontar essa pretensão.

A análise do tema das doenças negligenciadas e sua inserção no ensino por meio do exame das propostas curriculares é um esforço necessário e apresenta considerações importantes para que se repensem as ações desencadeadas no espaço escolar. Para uma melhor compreensão de como procede à dinâmica do tema das doenças negligenciadas nas aulas de Ciências é importante que esta observação não se limite ao currículo escrito e inclua-se o currículo ativo evitando, assim, distorções sobre uma possível dicotomia existente entre a teoria e a prática.

\section{REFERENCIAS BIBLIOGRÁFICAS}

AAGAARD-HANSEN, J.; CHAIGNAT, C.L. Neglected tropical diseases: equity and social determinants. In: Blas, E.; Kurup, A. S. (Org). Equity, social determinants and public health programmes. Genebra: Ed. WHO, 2010, p. 135-157. Disponível em: http://whqlibdoc.who.int/publications/2010/9789241563970_eng.pdf. Acesso realizado em: 7 de fevereiro de 2014.

ALVES-MAZZOTTI, A. J.; GEWANDSZNAJDER, F. O método nas Ciências naturais e sociais: pesquisa qualitativa e quantitativa. São Paulo: Pioneira, 1998.

APPLE, Michael. Repensando ideologia e currículo. In: MOREIRA, A. F. B.; SILVA, T. T. Currículo, cultura e sociedade. 6. ed. São Paulo: Cortez, 2002a, p.39-57

APPLE, Michael W. Podem as pedagogias críticas sustar as políticas de direita?. Caderno de Pesquisa, São Paulo, n.116, p. 107-142, 2002 b.

APPLE, Michael W. Ideologia e currículo. 3.ed. Porto Alegre; Artmed, 2006.

ARROYO, M.G. A função social do ensino de Ciências. Em Aberto, Brasília, v. 7, n. 40, 1988.

ASSIS, S. S.; PIMENTA, D. N.; SCHALL, V. T. Conhecimentos e práticas educativas sobre dengue: a perspectiva de professores e profissionais de saúde. Ensaio: Pesquisa em educação em ciências, Belo Horizonte, v. 15, n. 1, $2013 \mathrm{a}$. 
ASSIS, S. S.; PIMENTA, D. N.; SCHALL, V. T. A dengue nos livros didáticos de ciências e biologia indicados pelo Programa Nacional do Livro Didático. Ciência e educação, Bauru, v. 19, n.3, 2013b.

BRASIL. Departamento de Ciência e Tecnologia, Secretaria de Ciência, Tecnologia e Insumos Estratégicos, Ministério da Saúde. Doenças negligenciadas: estratégias do Ministério da Saúde. Revista de Saúde Pública, São Paulo, v. 44, n. 1, p. 200-202, 2010.

BRASIL. SINAN - TabNet. Indicadores e dados básicos - Brasil- 2012. IDB-2012. Disponível em: http://dtr2004.saude.gov.br/sinanweb/. Acesso realizado em: $14 \mathrm{de}$ janeiro de 2014.

BRITO, L. D.; FREITAS, D. O ensino de Biologia e as possibilidades de construções curriculares pela comunidade escolar: o caso da disciplina Biologia Aplicada. In: In: Teixeira, P. M. M.; Razera, J. C. C. (Org.). Ensino de Ciências: pesquisas e pontos em discussão. Campinas: Komedi, 2009. p. 191-226.

FRANÇA, V. H. As Leishmanioses em escolas do ensino básico de Divinópolis, MG: análise de livros didáticos de ciências e biologia e das representações sociais de professores sobre o tema. 2011. 217f. Dissertação (Mestrado em Ciências da Saúde) Instituto René Rachou, Fundação Oswaldo Cruz, Belo Horizonte, 2011.

FRANÇA, V. H. ; MARGONARI, C. ; SCHALL, V.T. . Percepção de professores do Ensino Básico em relação as suas práticas educativas sobre Leishmanioses: um estudo em área endêmica de Minas Gerais. Ensaio: Pesquisa em Educação em Ciências, Belo Horizonte, v. 15, p. 35-51, 2013.

GOODSON, I. F. Currículo, teoria e história. Petrópolis: Vozes, 1995.

INSTITUTO OSWALDO CRUZ (IOC/Fiocruz-RJ). Nota técnica nº 1/2011/IOCFiocruz/Diretoria. Disponível em:

http://www.fiocruz.br/ioc/media/NotaTecnica_1_2011_IOCatual.pdf. Acesso realizado em: 4 de junho de 2012.

KRASILCHIK, M. Reformas e realidade o caso do ensino das ciências. São Paulo em perspectiva. São Paulo, v.14: 85-93, 2000.

KRASILCHIK, M.; MARANDINO, M. Ensino de ciências e cidadania. 2 ed. São Paulo: Moderna, 2007.

LOPES, A. C. Reflexões sobre currículo: as relações entre senso comum, saber popular e saber escolar. Em Aberto, Brasília, v. 12, n.58, 1993.

MÉDICOS SEM FRONTEIRAS (MSF). Fatal Imbalance: The Crisis in Research and Development for Drugs for Neglected Diseases. Access to Essential Medicines Campaign and the Drugs for Neglected Diseases Working Group. Brussels: Médecins Sans Frontières. Geneva: Médicos Sem Fronteiras, 2001. 
MOHR, A. A natureza da educação em saúde no ensino fundamental e os professores de ciências. 2002. 410f. Tese (Doutorado em Educação) - Universidade Federal de Santa Catarina, Santa Catarina, 2002.

MOHR, A. A educação em saúde na escola: panorama e questionamentos a partir de depoimentos de professores de Ciências de Florianópolis. In: Selles, S. E.; Ferreira, M. S.; Barzano, M. A. L.; Silva, E. P. Q. Ensino de Biologia: histórias, saberes e práticas formativas. Uberlândia: EDUFU, 2009. p. 107 - 126.

ORGANIZAÇÃO DAS NAÇÕES UNIDAS (ONU). Declaração do Milénio. Nova Iorque: ONU, 2000. Disponível em:

http://www.unric.org/html/portuguese/uninfo/DecdoMil.pdf. Acesso realizado em: 21 de janeiro de 2014.

ORGANIZAÇÃO MUNDIAL DE SAÚDE (OMS). Macroeconomics and Health: Investing in Health for Economic Development. Geneva: WHO Publication, 2001.

REZENDE, F.; LOPES, A. M. A.; EGG, J. M. Identificação de problemas do currículo, do ensino e da aprendizagem de física e de matemática a partir do discurso de professores. Ciência e Educação, Bauru, v. 10, n.12, 2004.

SCHALL, V. T. Saúde e cidadania. In: Pavão, A. C. Ciências: ensino fundamental. Brasília: Ministério da Educação, Secretaria de Educação Básica, 2010. (Coleção Explorando o Ensino; v. 18). p. 179-196.

SEVERINO, A. J. Metodologia do trabalho científico. 20. ed. São Paulo: Cortez, 1996.

SILVA, T. T. Documentos de identidade: uma introdução às teorias do currículo. Belo Horizonte: Autêntica, 1999.

VENTURINE, T.; MOHR, A. Análise da Educação em Saúde nos Parâmetros Curriculares Nacionais a partir de uma nova perspectiva. In: ENCONTRO NACIONAL DE PESQUISAS EM EDUCAÇÃO EM CIÊNCIAS, 9, Águas de Lindóia: 10 -14 de novembro de 2013. Atas do IX Encontro Nacional de Pesquisas em Educação em Ciências. Águas de Lindóia: Associação Brasileira de Pesquisa em Educação em Ciências, 2013. Disponível em http://www.adaltech.com.br/sigeventos/abrapec2013/inscricao/resumos/0001/R00511.PDF . Acesso realizado em 08 de janeiro de 2014.

VICTORA, C. G.; KNAUTH, D. R.; HASSEN, M. N. A. Pesquisa qualitativa em saúde: uma introdução ao tema. Porto Alegre: Tomo Editorial, 2000. 